\title{
Possible link between congenital uterine malformations and epitheliod trophoblastic tumor
}

\author{
Balint Farkas $^{1 *}$, Krisztina Kovacs ${ }^{2}$, Miklos Vizer ${ }^{1}$, Jozsef Bodis ${ }^{1}$, Peter Gocze ${ }^{1}$ \\ ${ }^{1}$ Department of Obstetrics and Gynecology, University of Pecs Medical Center, Pécs, Hungary \\ ${ }^{2}$ Department of Pathology, University of Pecs, Pécs, Hungary \\ Email: dr.balint.farkas@gmail.com
}

Received 19 January 2012; revised 20 March 2012; accepted 7 April 2012

\begin{abstract}
Background: Epitheloid trophoblast tumor (ETT) is a tenuous type of gestational trophoblastic disease. Although clinically ETT behave in variable fashion, it is considered as a potentially malignant tumor, fatal malignancy occurs in $10 \%$ of the cases. Case and Conclusion: A 41 years old patient, who previously underwent Tompkins operation because of congenital uterine septum was admitted to our clinic due to irregular bleeding. Despite of serum hCG levels under the upper limit of normal, histopathology analysis of D\&C obtained endometrial samples confirmed the presence of ETT by immunostainings. Henceforth we performed prompt abdominal hysterectomy and preserved the normal structured ovaries. According to the müllerianosis theory of endometriosis aberrant differentiation or migration could cause spreading of cells throughout the fetal organogenesis of the uterus. These cells in an apoptosis week environment might correlate with the initiation of ETT. As a conclusion we suggest further studies to assess the link between ectopic cells and ETT, to gain a better understanding in the pathomechanism of the tumor.
\end{abstract}

Keywords: Epitheloid Trophoblastic Tumor; Uterine Septum; Congenital Uterine Malformation; Intermediate Trophoblast

\section{INTRODUCTION}

Gestational trophoblastic neoplasm (GTN) is a group of rare tumors which involve abnormal proliferation of trohoblast cells inside the uterus during or after pregnancy [1]. Recent clinicopathological investigations reported three distinctive types: 1) choriocarcinoma, 2) placental site trophoblastic tumor (PSTT), and 3) epitheloid trophoblastic tumor (ETT). The clinico-pathologic characteristics of ETT were first described by Shih and Kurman

*Corresponding author. thirteen years ago [2], since then nearly 19 Englishlanguage papers reported 50 cases of ETT. It is proposed to be origin from chorionic-type extravillous (intermediate) cytotrophoblast cells, which are normally seen in the chorion leave (fetal membranes) [2,3] (Figure 1).

The clinical features and diagnosis shows wide spectrum of variability ranging from benign type, remaining in the uterine cavity, to highly malignant type resulting widespread metastases (25\%) or even fatal outcome $(10 \%)$ [4]. It usually occurs in woman at their reproductive age followed by a normal pregnancy, or elective abortion or mole, with a symptom of irregular vaginal bleeding.

\section{CASE REPORT}

A 41 years old patient was admitted to our clinic, complaining about irregular vaginal bleeding and lower abdominal pain with left side preponderance. Medical history contained three consecutive spontaneous abortions followed by a Tompkins metroplasty, due to the presence of a partial uterine septum dividing the uterine fundus.

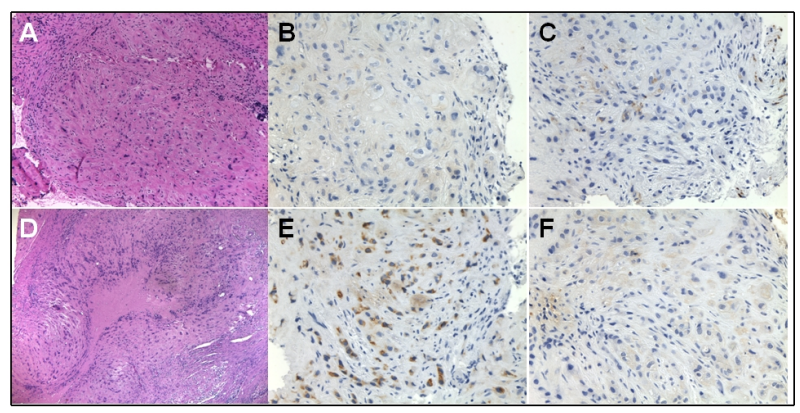

Figure 1. Characteristic features of epitheloid trophoblastic tumor. Group of tumor cells from endometrial curettage (D \& C) have abundant cytoplasm and irregular hyperchromatic nuclei resulting epitheloid appearance (A) (H \& E ×100). Note geographical necrosis within the residual tumor (D) $(\mathrm{H} \& \mathrm{E} \times 40)$. Immunoprofile of trophoblastic tumor cells shows negativity for hCG (B) and Placental Alkaline Phosphatase (C), mostly negativity, but focally positivity for $\mathrm{hpL}(\mathrm{F})$ and intense diffuse positivity for Inhibin $(E)(\times 200)$. 
After the operation she delivered a healthy infant via caesarian section in 1997 and she interrupted her last pregnancy seven years ago. A D \& C was carried out and histopathology diagnosed persistent gestational trophoblastic tumor, with epitheloid origin. Diagnosis was confirmed by immunhistochemistry, immunostaining of the tumor showed inhibin and cytokeratin positivity, in contrast human chorionic gonadotropin (hCG) and human placental lactogen $(\mathrm{hpL})$ negativity.

Pregnancy test was found to be negative, serum betahCG was very low (!): $0.56 \mathrm{IU} / \mathrm{L}$, it was under the upper limit of normal. Gynecological ultrasound examination described a normal sized uterus with increased vascularrity on the right side of the fundus, but no pathological blood flow or any signs of neovascularization. Endometrium thickness was $8 \mathrm{~mm}$, with edgy endomyometrial border. Both ovaries were measured normal sized and structured. Staging, utilizing two bearing oriented chest $\mathrm{X}$-ray and pelvic and abdominal MRI scan, appointed that the disease is currently restricted to the uterus. Considering the results, without delay abdominal hysterictomy was carried out when both the ovaries were conserved. Histopathology confirmed the presence of ETT. Patient was discharged from the hospital in good general condition and was instructed to participate in the close, periodic follow up and until current date no signs of recurrence have been observed.

\section{DISCUSSION}

In embryonic life the paramesonephric ducts are merging to develop the uterus. Through a controlled apoptosis executed by the Bcl-2 proteins are suggested to be responsible for the regression of the midline, result the normal shape of the uterus [5]. Although the organogenesis is still not completely understood, the key role of anti-Müllerian hormone signaling pathway is suspected [6]. The müllerianosis theory of the pathogenesis of endometriosis discusses aberrant differentiation or migration of the Müllerian ducts, which could cause spreading of cells or tracts of cells in the migratory pathway of the fetal organogenesis of the uterus. These ectopic cells in a lack of apoptosis environment could trigger the transformation into neoplastic cells. Further studies are urgently required to assess the presence of such ectopic cells to gain a better understanding in the pathomechanism of ETT [7].

Although elevated serum hCG levels are reported widely in ETT, the result can vary and often can be low, moreover generally does not exceed $2.500 \mathrm{IU} / \mathrm{L}$ [8], in contrast to choriocarcinoma [9]. Serum hCG shows variability in some of the cases, the values shows low-level elevation, which possibly reflect to the lack of syncytiotrophoblast in this neoplasia. A recent study highlighted that $14 \%$ of the reported cases had hCG levels $<2.0 \mathrm{IU} / \mathrm{L}$, but $25 \%$ of cases had levels over $150.000 \mathrm{IU} / \mathrm{L}[8,9]$. Therefore it is very important to consider ETT as a diagnosis even when the serum hCG levels are negative or high, furthermore the serum level of hCG in ETT, unlike in choriocarcinoma, is not an accurate indicator of the tumor and can not be used as a reliable tumor marker. Till date it is frequently debated and still unclear, that serum hCG levels can help to predict the outcome of ETT, although some authors suggests to determine the serum levels of inhibin, which can be useful in the oncology follow up procedure by monitoring and detecting recurrences [2]. Precaution has to be done though, because hormone producing ovarian tumors, such as granulose cell ovarian tumor, also have high level of serum inhibin levels [10].

Based on earlier studies, which demonstrated that neither the histopathologic features, such as the tumor size, or the percentage of necrosis or cytologic atypia, nor the immunohistochemical features correlates with the tumor behavior [2,8,11], and despite the relatively low malignancy ratio (only $10 \%$ of ETT has a clinically malignant characteristic) our group recommends radical hysterictomy without further delay, especially in women in the age of 40 or over, unless the preservation of fertility is an important factor. In the early stage when the tumor is restricted to the uterus hysterectomy is the optimal therapy and the ovaries may be preserved if they appear macroscopically normal $[12,13]$. The necessity of pelvic or paraaortic lymphadenectomy is controversial.

Unfortunately ETT does not respond to chemotherapeutic agents used in the treatment of other types of gestational trophoblast disease $[14,15]$. Therefore combination chemotherapy, which was found to be highly effective in PSTT, such as EMA/CO utilizing Etoposide, Methotrexte, Dactinomycin alternating with Cyclophosphamide and Vincristine, or EMA/EP (when Cisplatin is added to the regimen in refractory cases), shows minimal results.

\section{CONCLUSION}

The clinical and therapeutic implication of this case report is straightforward. ETT should be regarded as a potentially metastatic malignant tumor, therefore complete surgery is considered to be curative. It must be underlined that D \& C sampling, instead of the use of pipelle is the proper way to diagnose such malignancies. In patients with extensive or metastatic disease reductive surgery or palliative chemotherapy should be chosen.

\section{REFERENCES}

[1] Seckl, M.J., Sebire, N.J. and Berkowitz, R.S. (2010) 
Gestational trophoblastic disease. Lancet, 376, 717-729. doi:10.1016/S0140-6736(10)60280-2

[2] Shih, I.M. and Kurman, R.J. (1998) Epithelioid trophoblastic tumor: A neoplasm distinct from choriocarcinoma and placental site trophoblastic tumor simulating carcinoma. The American Journal of Surgical Pathology, 22, 1393-1403. doi:10.1097/00000478-199811000-00010

[3] Shih, I.M. (2007) Gestational trophoblastic neoplasiapathogenesis and potential therapeutic targets. Lancet Oncology, 8, 642-650.

doi:10.1016/S1470-2045(07)70204-8

[4] Kimberly, H., Allison, M.D., Jason, E., Love, M.D., Rochelle, L. and Garcia, M.D. (2006) Epithelioid trophoblastic tumor: Review of a rare neoplasm of the chorionic-type intermediate trophoblast. Archives of $\mathrm{Pa}$ thology \& Laboratory Medicine, 130, 1875-1877.

[5] Lee, D.M., Osathanondh R. and Yeh, J. (1998) Localisation of Bcl-2 in the human fetal müllerian tract. Fertil Steril, 70, 135-140. doi:10.1016/S0015-0282(98)00126-5

[6] Klattig, J. and Englert, C. (2007) The Müllerian duct: Recent insight into its development and regression. Sexual Development, 1, 271-278. doi:10.1159/000108929

[7] Mai, K.T., Yazdi, H.M., Perkins, D.G. and Parks, W. (1998) Development of endometriosis from embryonic duct remnants. Human Pathology, 29, 319-322. doi:10.1016/S0046-8177(98)90110-1

[8] Palmer, J.E., Macdonald, M., Wells, M., Path, F.R.C., Hancock, B.W. and Tidy, J.A. (2008) Epitheliod trophoblast tumor. A review of the Literature. The Journal of
Reproductive Medicine, 53, 465-475.

[9] Allison, K.H., Love, J.E. and Garcia, R.L. (2006) Epitheloid trophoblastic tumor: Review of a rare neoplasm of the chorionic type intermediate trophoblast. Archives of Pathology \& Laboratory Medicine, 130, 1875-1877.

[10] Fadare, O., Parkash, V., Carcangiu, M.L., et al. (2006) Epitheloid trophoblast tumor: Clinicopathologic features with an emphasis on uterine cervical involvement. Modern Pathology; 19, 75-82. doi:10.1038/modpathol.3800485

[11] Gocze, M.P., Beamer, G.W., De Jong, H.F. and Freeman, A.D. (1997) Hormone synthesis and responsiveness of spontaneous granulose cell tumors in $(\mathrm{SWR} \times \mathrm{SWXJ}-9)$ F1 mice. Gynecology Oncology, 65, 143-148. doi:10.1006/gyno.1997.4635

[12] Ajithkumar, T.V., Abraham, E.K., Rejnishkumar, R. and Minimole, A.L. (2003) Placental site trophoblastic tumor. Obstetrical \& Gynecological Survey, 58, 484-488. doi:10.1097/01.OGX.0000077466.40895.32

[13] Driscoll, E.G. (1984) Placental site chorioma, the neoplasm of the implantation site trophoblast. The Journal of Reproductive Medicine, 29, 821-825.

[14] Knox, S., Brooks, S.E., et al. (2002) Choriocarcinoma and epithelial trophoblastic tumor: Successful treatment of relapse with hysterectomy and high-dose chemotherapy with peripheral stem cell support: A case report. Gynecologic Oncology, 85, 204-208.

[15] Kurman, R.J. (2002) Blaustein's pathology of the female genital tract. 5th Edition, Springer, New York. 